\title{
Use of Three-Dimensional Printing in Modelling an Anatomical Structure with a High Computed Tomography Attenuation Value: A Feasibility Study
}

\author{
Lakna N. Kariyawasam ${ }^{1,2}$, Curtise K. C. Ng ${ }^{1, *}$, Zhonghua Sun ${ }^{1}$, and Catherine S. Kealley 1,3,4 \\ ${ }^{1}$ Discipline of Medical Radiation Science, School of Molecular and Life Sciences, Curtin University, Perth, \\ Western Australia 6845, Australia \\ ${ }^{2}$ Imaging Services, Royal Perth Hospital, Wellington Street, Perth, Western Australia 6000, Australia \\ ${ }^{3}$ Department of Physics, The University of Western Australia, 35 Stirling Highway, Crawley, Western Australia 6009, Australia \\ ${ }^{4}$ Department of Medical Imaging and Radiation Sciences, Monash University, Wellington Road, Clayton, Victoria 3800, Australia
}

\begin{abstract}
Introduction: Three-dimensional (3D) printing provides an opportunity to develop anthropomorphic computed tomography (CT) phantoms with anatomical and radiological features mimicking a range of patients' conditions, thus allowing development of individualised, low dose scanning protocols. However, previous studies of 3D printing in CT phantom development could only create anatomical structures using potassium iodide with attenuation values up to $1200 \mathrm{HU}$ which is insufficient to mimic the radiological features of some high attenuation structures such as cortical bone. This study aimed at investigating the feasibility of using 3D printing in modelling cortical bone with a non-iodinated material. Methods: This study had 2 stages. Stage 1 involved a vat photopolymerisation 3D printer to directly print cube phantoms with different percentage compositions of calcium phosphate (CP) and resin (approach 1), and approach 2 using a material extrusion 3D printer to develop a cube mould for infilling of the CP with hardener as the phantom. The approach able to create the cube phantom with the CT attenuation value close to that of a tibial mid-diaphysis cortex of a real patient, $1475 \pm 205 \mathrm{HU}$ was employed to develop a tibial mid-diaphysis phantom. The mean CT numbers of the cube and tibia phantoms were measured and compared with that of the original CT dataset through unpaired $t$-test. Results: All phantoms were scanned by CT using a lower extremity scanning protocol. The moulding approach was selected to develop the tibia middiaphysis phantom with CT attenuation value, $1434 \pm 184 \mathrm{HU}$ which was not statistically significantly different from the one of the original dataset $(p=0.721)$. Conclusion: This study demonstrates the feasibility to use the material extrusion 3D printer to create a tibial mid-diaphysis mould for infilling of the $\mathrm{CP}$ as an anthropomorphic CT phantom and the attenuation value of its cortex matches the real patient's one.
\end{abstract}

Keywords: Calcium Phosphate, Computed Tomography, Hounsfield Unit, Mould, Phantom, Three-Dimensional Printing.

\section{INTRODUCTION}

Use of three-dimensional (3D) printing in medicine has become popular for some years [1]. Examples of its applications include development of personalised medical devices and anatomical models for surgical planning, teaching and research [1-7]. Recently, studies have shown the use of 3D printing in anthropomorphic phantom development for quality assurance of medical radiation sciences procedures such as intensity modulated radiation therapy $[8,9]$, stereotactic body radiation therapy [10], paediatric radiography [11], digital tomosynthesis [12-14] and computed tomography (CT) [2,15-18]. Among those studies, the

*Author to whom correspondence should be addressed.
CT phantom development appears to be the more common 3D printing research area.

The $\mathrm{CT}$ is the greatest contributor to collective medical radiation dose. However, CT scanning protocols of many institutions are not optimised as per patients' characteristics to address its radiation dose burden $[19,20]$. Commercial phantoms have been used for CT scanning protocol optimisation for decades [18]. Despite their high cost, they only mimic features of standardsized patients which appear not suitable for testing customised scanning protocols for various patients' characteristics, e.g., individual patients' sizes, pathological conditions, etc. Their use for the CT dose optimisation tends to be limited [2, 4, 17, 18]. Nonetheless, the 3D printing provides an opportunity to develop the CT phantoms with anatomical and radiological features 
mimicking a range of patients' conditions which allow development of individualised, low dose protocols to suit different clinical tasks without sacrificing diagnostic quality [16-18]. Also, their development process requires shorter time with lower cost when compared to the commercial phantoms [3, 17, 18].

The technologies for $3 \mathrm{D}$ printing in medicine include vat photopolymerisation (stereolithography [SLA]/digital light processing [DLP]), material jetting (PolyJet/multi-jet modelling $[\mathrm{MJM}]$ ), binder jetting, material extrusion (fused deposition modelling $[\mathrm{FDM}]$ ), powder bed fusion (selective laser sintering [SLS]/direct metal laser sintering [DMLS]/selective laser melting $[\mathrm{SLM}] /$ electron beam melting $[\mathrm{EBM}]$ ), sheet lamination and directed energy deposition. Their details including advantages and limitations have been published elsewhere [5, 21], and only the key issues related to the CT phantom development are discussed in this paper.

The previous CT phantom development studies reported uses of the material jetting to construct lung [16], liver and brain phantoms [17], the material extrusion to build a cardiac model [2], and the sheet lamination to produce abdomen [18], head and neck phantoms [15]. Those studies claimed their CT phantoms had shapes and attenuation values measured in Hounsfield unit (HU)/CT numbers similar to corresponding anatomical structures. However, apparently, their phantom evaluation just covered the CT attenuation value measurement because the aim of their phantom development was for CT scanning protocol optimisation rather than surgical planning which requires a close resemblance of shape [2, 15-18]. It is noted that the traditional 3D printing materials such as photopolymers can only produce models with CT numbers about $20-130 \mathrm{HU}$ and are unable to make bone-mimicking structures. In order to produce structures with high attenuation values, substances such as iodine, barium, calcium and bismuth can be mixed with the traditional materials for $3 \mathrm{D}$ printing $[16,17]$. Another strategy is to use the traditional resins to make a 3D printed container/mould resembling an anatomical structure with infill of the high attenuation materials to form a phantom. The mould could be removed after infilling if required [17]. The former approach was used by Jahnke et al. [18] to radiopaquely print stacks of CT images using mixtures of standard inkjet ink and potassium iodide on paper for creating the CT phantoms via the sheet lamination method. Their phantoms had attenuation values up to $1200 \mathrm{HU}$. The latter approach was successfully implemented by Abdullah et al. [2] to produce the cardiac phantom with hollow regions for filling air (-894.1 HU), oil (-92.4 HU), jelly (25.9 HU) and iodinated contrast medium (354.3 HU).

The potassium iodide appears as a promising material to create individualised CT phantoms of high attenuation structures with CT numbers up to $1200 \mathrm{HU}[2,18]$. However, the other materials for this purpose suggested in the literature seem not tested yet [17]. The common high attenuation structure in human body is cortical bone with CT numbers ranging between $500 \mathrm{HU}$ and $2000 \mathrm{HU}[22,23]$. To fully realise the use of 3D printing in development of customised CT whole body phantom which has structures with attenuation values ranging between $-1000 \mathrm{HU}$ and $2000 \mathrm{HU}$ for CT scanning protocol optimisation, future studies should focus on cortical bone model development as this remains a challenging area $[16,22]$. The aim of this study was to investigate the feasibility of using 3D printing in modelling the cortical bone with a non-iodinated material.

\section{METHODS}

This study's design was similar to the other 3D printing study in the CT phantom development which had two stages [18]. Stage 1 involved creation of $2 \mathrm{~cm}^{3}$ cube phantoms with various attenuation properties to identify appropriate arrangements for stage 2 to develop a CT anthropomorphic phantom. The aforementioned two approaches for making high attenuation structures were implemented in stage 1 [17]. The anthropomorphic phantom developed in stage 2 was a model of a right tibial mid-diaphysis of a 70-year-old male patient who underwent a CT scan (without any remarkable abnormality). The tibial middiaphysis was chosen for the phantom development because it has high cortical to trabecular bone ratio and simple geometry suitable for this study [24, 25]. A free, open source 3D visualisation program, 3DSlicer 4.8.1 (The Slicer Community, MA, USA) was used with its 'draw a 3D cube' tool to make the 3D cube mesh model, and for image post-processing and segmentation of anonymised digital imaging and communications in medicine (DICOM) CT images of the patient to make the tibia and fibula mesh model based on the segmented volume data. The mesh models were saved in standard tessellation language (STL) format for 3D printing [2, 7-9, 21]. Institutional review board approval was waived because of the use of the anonymised CT images. The high attenuation material selected for the phantom development was calcium phosphate (CP) monobasic monohydrate $\left(\mathrm{Ca}\left(\mathrm{H}_{2} \mathrm{PO}_{4}\right)_{2} \cdot \mathrm{H}_{2} \mathrm{O}\right)$ as the $\mathrm{CP}$ is the major component of the cortical bone [26].

\subsection{Stage 1-Cube Phantom Development}

For the first approach, a vat photopolymerisation 3D printer (Kudo3D Titan 1, Kudo3D Inc., CA, USA) was used with mixtures of photocurable liquid resin (Monocure 3D Photoreactive Clear Resin, Monocure Pty Ltd., NSW, Australia) and the ring milled $\mathrm{CP}$ to create the cube phantoms with different percentage compositions of these two materials $[5,17]$. The vat photopolymerisation 3D printer is commonly used to directly print bone models because it can produce models with fine details [5, 27]. Ring milling of the $\mathrm{CP}$ was to reduce its particle size for facilitating the mixing process [28].

Similar to Abdullah et al.'s study [2], a material extrusion 3D printer (Axiom 20, Airwolf 3D, CA, USA) was employed to implement the second approach by creating a cube container (mould) using NinjaFlex 3D printing material (Fenner Inc., PA, USA) for infilling of a $2 \mathrm{~cm}^{3}$ volume of $\mathrm{CP}$ and epoxy resin (hardener) mixture. The main advantage of the material extrusion $3 \mathrm{D}$ printer is its low cost $[5,27]$. The mixture was prepared by gradually adding the epoxy resin and its hardener to the $\mathrm{CP}$ with stirring until becoming a paste. After the mixture hardened (48 h), the mould was cut out to form the cube phantom [29]. Figure 1 summarises the steps of approaches 1 and 2 .

\subsection{Stage 2-Tibial Mid-Diaphysis Phantom Development}

The approach able to create the cube phantom with the CT attenuation value close to that of the right tibial mid-diaphysis cortex of the original CT dataset, $1475 \pm 205 \mathrm{HU}$ (measured over 6 consecutive axial images) was employed to create the right tibial mid-diaphysis phantom [15]. A free, open source mesh processing program, MeshLab 2016.12 (Istituto di Scienza e Tecnologie dell'Informazione, Pisa PI, Italy) was used to refine the mesh 


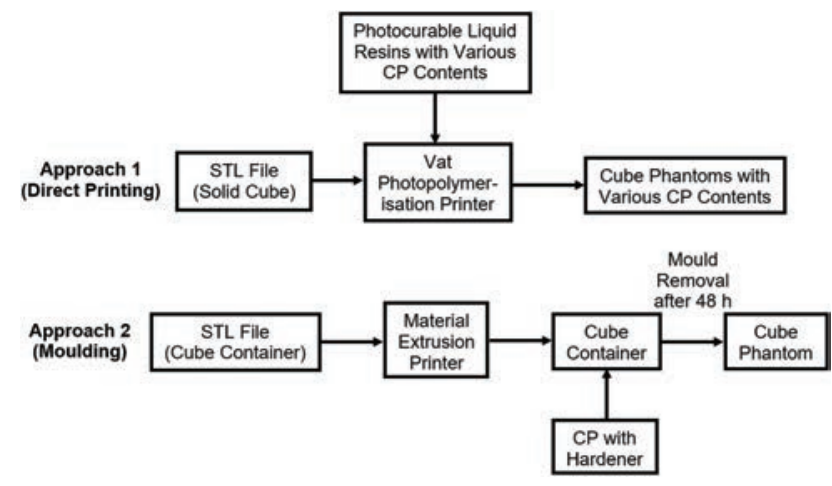

Fig. 1. Summary of the steps involved in approaches 1 and 2 of stage 1 . $\mathrm{CP}$, calcium phosphate; STL, standard tessellation language.

model generated by the 3DSlicer 4.8 .1 program prior to the $3 \mathrm{D}$ printing [30].

\subsection{Evaluation of $\mathrm{CT}$ Attenuation Property of Phantoms}

Both the cube and tibial mid-diaphysis phantoms were scanned on a 128-slice CT scanner (SOMATOM Definition AS+, Siemens Medical Solutions, PA, USA) with following scanning parameters, $100 \mathrm{kV}, 100 \mathrm{~mA}, 2 \mathrm{~mm}$ slice thickness and 0.8 pitch similar to those used for the original lower extremity CT dataset acquisition. The acquired DICOM CT images of the phantoms were exported to a workstation with a free, open source image processing program, ImageJ 1.47 (National Institutes of Health, MD, USA) for measuring average CT attenuation values of regions of interest (the cube and the tibial mid-diaphysis cortex) over a volume of 6 consecutive axial images [15]. An unpaired $t$-test was used to compare the average CT attenuation value of each phantom with that of the right tibial mid-diaphysis cortex of the original CT dataset. GraphPad InStat 3.06 (GraphPad Software Inc, CA, USA) was used for the statistical analysis. A $p$-value less than 0.05 obtained from the $t$-test was considered statistically significant difference.
Table I. The computed tomography (CT) attenuation values of the cube phantoms.

\begin{tabular}{lcc}
\hline Cube (composition) & $\begin{array}{c}\text { CT attenuation value } \\
\text { (Mean } \pm \text { SD) }\end{array}$ & $P$-value* \\
\hline A ( $100 \%$ of photocurable & $109 \pm 20 \mathrm{HU}$ & $<0.0001$ \\
$\quad$ liquid) & $179 \pm 47 \mathrm{HU}$ & $<0.0001$ \\
$\begin{array}{l}\text { B }(90 \% \text { of photocurable liquid } \\
\quad \text { with } 10 \% \text { of } \mathrm{CP})\end{array}$ & $195 \pm 82 \mathrm{HU}$ & $<0.0001$ \\
$\begin{array}{l}\quad(80 \% \text { of photocurable liquid } \\
\text { with } 20 \% \text { of CP) }\end{array}$ & $474 \pm 105 \mathrm{HU}$ & $<0.0001$ \\
$\begin{array}{l}\text { D (70\% of photocurable liquid } \\
\quad \text { with } 30 \% \text { of } \mathrm{CP})\end{array}$ & $1493 \pm 72 \mathrm{HU}$ & 0.8459 \\
\hline E (CP with hardener) &
\end{tabular}

Notes: CP, calcium phosphate; $\mathrm{HU}$, Hounsfield unit; SD, standard deviation. ${ }^{*} P$-value of unpaired $t$-test for comparing the mean CT attenuation value of each phantom with that of the tibial mid-diaphysis cortex of the original CT dataset, $1475 \pm 205 \mathrm{HU}$.

\section{RESULTS}

\subsection{Stage 1-Cube Phantom Development}

Figure 2 illustrates the cube phantoms developed by using the approach 1-direct printing (cubes A-D) and the approach 2-moulding (cube E), and their corresponding axial CT images. An irregular contour is noted in the cube D images because of excessive $\mathrm{CP}$ within the resin $(70 \%$ of photocurable liquid with $30 \%$ of $\mathrm{CP}$ ) causing it unable to become a proper colloidal suspension for the vat photopolymerisation $3 \mathrm{D}$ printing. Resins with the CP content greater than $30 \%$ were not prepared and tested further. The cube $\mathrm{E}$ was created through putting the $\mathrm{CP}$ with hardener into the cube mould printed by the material extrusion 3D printer. The cube E appears not accurately resembling the shape of a cube. Table I shows the CT attenuation values of these cube phantoms. Only the CT attenuation value of the cube E matches the one of the tibial mid-diaphysis cortex of the original CT dataset. The moulding approach was used in stage 2 for the tibial mid-diaphysis phantom development.

\subsection{Stage 2-Tibial Mid-Diaphysis Phantom Development}

Figure 3 illustrates the mould printed by the material extrusion $3 \mathrm{D}$ printer and infilling of the $\mathrm{CP}$ with hardener to create the

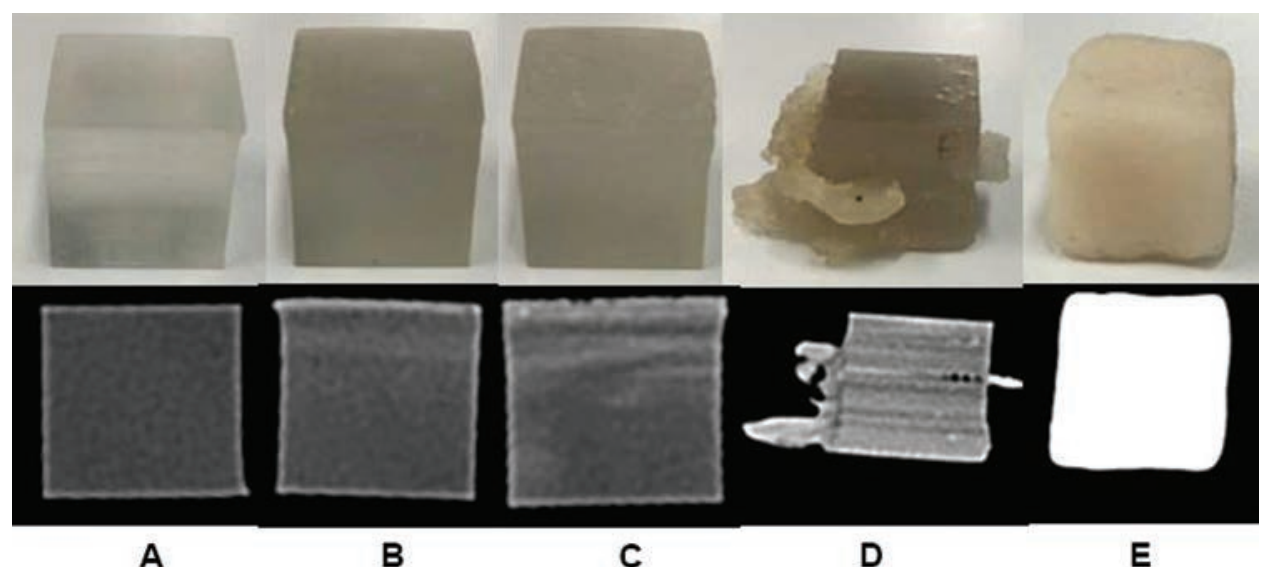

Fig. 2. The cube phantoms developed with the use of the approach 1-direct printing (cubes A-D) and the approach 2-moulding (cube E), and their corresponding axial computed tomography images. The resin compositions of the cubes A-D were $100 \%$ of photocurable liquid, $90 \%$ of photocurable liquid with $10 \%$ of calcium phosphate (CP), $80 \%$ of photocurable liquid with $20 \%$ of $\mathrm{CP}$, and $70 \%$ of photocurable liquid with $30 \%$ of $\mathrm{CP}$ respectively. 

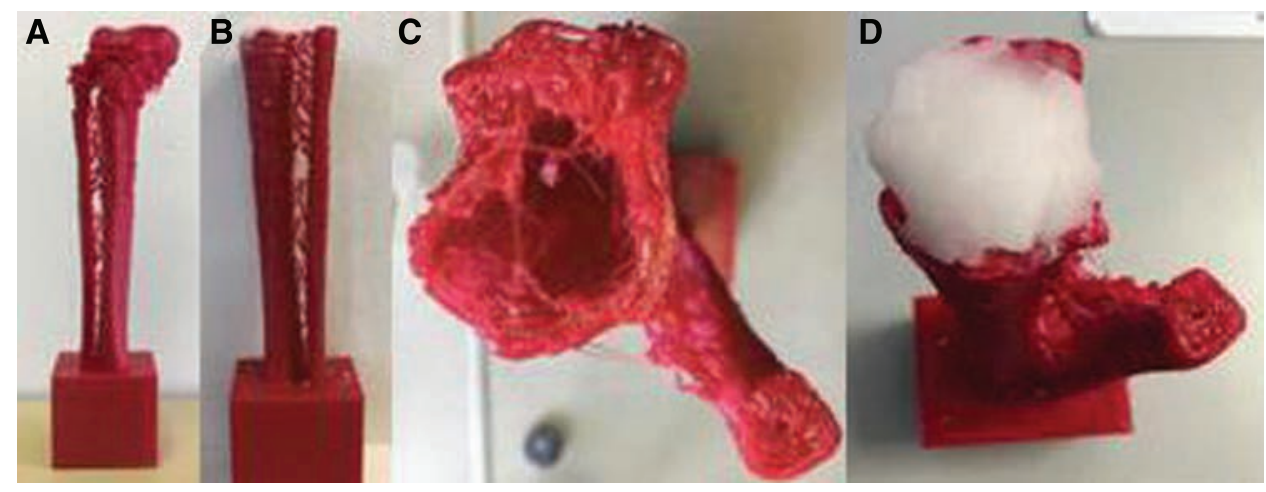

Fig. 3. The tibial mid-diaphysis phantom development process. (A) The mould was printed. (B and C) The mould with its proximal (top) part removed for infilling of the CP with hardener. (D) The mould with the infill of the CP.

tibial mid-diaphysis phantom. The mould printing time was about $6 \mathrm{~h}$, and the phantom hardening and mould removal process required $48 \mathrm{~h}$. The cost of the NinjaFlex 3D printing material and the CP were about US\$14 and US\$50 respectively. Figure 4 shows the tibial mid-diaphysis phantom and the original CT images. The phantom resembled the features of the tibial middiaphysis to some extent. For example, its anterior crest was illustrated. However, the contour of the phantom was not smooth. The CT attenuation value of the phantom was $1434 \pm 184 \mathrm{HU}$ which was not statistically significantly different from that of the original dataset $(p=0.721)$.

\section{DISCUSSION}

This study shows the material extrusion 3D printer could be used to create the tibial diaphysis mould for infilling of the $\mathrm{CP}$ and hardener mixture to develop the CT phantom of the cortical bone of the tibial mid-diaphysis with its attenuation value similar to that of the real patient, $1475 \pm 205$ HU. The highest attenuation value that could be achieved in the previous study of $3 \mathrm{D}$ printing in CT phantom development was $1200 \mathrm{HU}$. The potassium iodide was used as part of its 3D printing material [18]. Apparently, the use of potassium iodide may not be adequate to create some high attenuation anatomical structures but this is required for building a customised CT whole body phantom with the CT numbers of structures ranging between $-1000 \mathrm{HU}$ and $2000 \mathrm{HU}$ [16]. The CP could be utilised for developing these high attenuation structures.
The tibial mid-diaphysis phantom development time and cost of this study were comparable to the other similar study which were shorter and less expensive than those of the commercial phantom [2]. Although the general shape of the phantom appears similar to the original one, some external contour defects are also found (Fig. 4). As the NinjaFlex 3D printing material is not transparent, it was difficult to ensure the $\mathrm{CP}$ and hardener mixture filling up the mould completely causing the contour defects eventually. This issue was also reported in a study using a 3D printed mould to develop a kidney phantom. Use of transparent printing materials such as VeroClear and TangoPlus (Stratasys Ltd., MN, USA) with a material jetting printer was recommended. The TangoPlus is better for the mould creation because of its flexibility leading to ease of the mould removal [31]. However, these printing materials are relatively expensive [5].

The tibia mid-diaphysis phantom developed in this study also had another issue which was not a hollow structure. As noted in that kidney phantom development study, an outer and an inner moulds should be prepared for creating a phantom with a hollow space. Alignment of the 2 moulds involved in that approach might be challenging and their misalignment could affect geometrical accuracy of the phantom [31]. However, unlike the models for surgical planning, the anthropomorphic CT phantoms do not require high geometrical accuracy normally [2, 15-18]. The tibia mid-diaphysis phantom created in this study should serve its purpose to some extent.

The tibial mid-diaphysis was selected for the phantom development in this feasibility study because of its simple geometry [25].
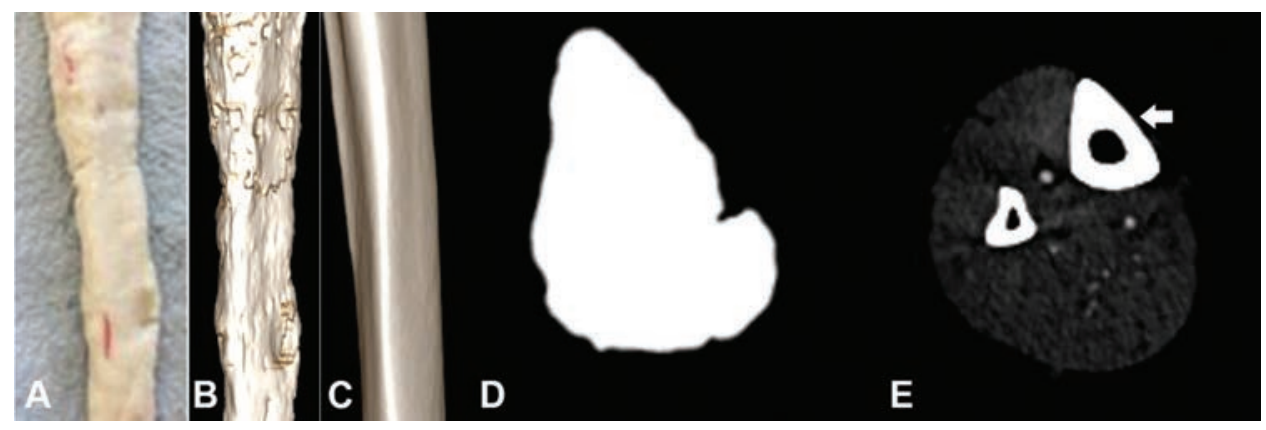

Fig. 4. The tibial mid-diaphysis phantom and original computed tomography (CT) images. (A) The photograph of the phantom. (B) The surface rendered CT image of the phantom. (C) The surface rendered CT image of the tibial mid-diaphysis of the original dataset. (D) The axial CT image of the phantom. (E) The axial CT image of the original dataset showing the tibial mid-diaphysis (arrow). 
Nevertheless, this phantom developed by the moulding approach still had a number of geometrical defects. It is noted in Figure 2 that the direct printing approach would be better to create phantoms with higher geometrical accuracy which is important for modelling complex anatomical structures such as molar teeth. A previous study demonstrated feasibility of mixing $15 \%$ of $\mathrm{CP}$ nanoparticles with $85 \%$ of thermoplastics for the material extrusion 3D printer to directly print a molar tooth model. However, its CT attenuation value was not assessed, thus unable to determine whether it was comparable to a real tooth's attenuation value [32]. This study shows that it was not feasible to use the vat photopolymerisation printer to directly print a cube phantom with high (30\% of) CP content. Nonetheless, it remains unclear whether models with CP content greater than $30 \%$ can be directly printed by the material extrusion and/or vat photopolymerisation printers when the $\mathrm{CP}$ nanoparticles are used. It is because $\mathrm{CP}$ particle size affects printability of the 3D printing materials [28].

This study has several limitations. Only two types of 3D printers (vat photopolymerisation and material extrusion) and one high attenuation material ( $\mathrm{CP}$ monobasic monohydrate) were involved in this study. However, most of the previous studies of $3 \mathrm{D}$ printing in the $\mathrm{CT}$ phantom development just used one single printer [2, 16-18]. Furthermore, it is not uncommon to investigate only one high attenuation material for the phantom creation $[15,18]$. The geometrical accuracy of the cube and tibial mid-diaphysis phantoms was not quantitatively assessed and the inner mould was not printed for creating a hollow tibial middiaphysis phantom. Nonetheless, high geometrical accuracy is normally not required for the anthropomorphic CT phantoms and hence this was not quantitatively evaluated in the previous studies either [2, 15-18]. For future research, more types of 3D printers $[5,21]$ and a wide range of high attenuation materials such as barium and bismuth [17] with different sizes including nanoparticles [32] as part of the printing materials should be investigated for developing phantoms with high attenuation structures. Apart from assessment of their CT attenuation properties, their geometrical accuracy should also be quantitatively assessed.

\section{CONCLUSION}

A tibial mid-diaphysis CT phantom with its attenuation value of the cortex $(1434 \pm 184 \mathrm{HU})$ matching the real patient's one ( $1475 \pm 205 \mathrm{HU})$ was developed by the moulding approach with the use of the material extrusion 3D printer and the CP. The attenuation value of this phantom is greater than the highest attenuation value (1200 HU) achieved in the previous study of 3D printing in the CT phantom development with the use of potassium iodide as part of the printing material. The CP seems to be a better choice to create the high attenuation structures such as the cortical bone for developing a customised CT whole body phantom.

The general shape of the tibial mid-diaphysis phantom appears similar to that of the original dataset. However, it had some external contour defects and was not a hollow structure. The direct printing approach would be better to create the phantoms with higher geometrical accuracy but mixing the high attenuation matter with the traditional printing materials to directly print the anatomical structures with high attenuation values remains a technical challenge which needs to be resolved. The use of the $\mathrm{CP}$ nanoparticles as the high attenuation material might address this issue.

\section{Conflicts of interest}

There are no conflicts to declare.

Acknowledgments: The authors would like to thank Mr. Ming Lim and Ms. Veronica Avery for their assistance in 3D printing. This study did not receive any specific grant from funding agencies in the public, commercial, or not-for-profit sectors.

\section{References and Notes}

1. Squelch, A., 2018. 3D printing and medical imaging. Journal of Medical Radiation Sciences, 65(3), pp.171-172.

2. Abdullah, K.A., McEntee, M.F., Reed, W. and Kench, P.L., 2018. Development of an organ-specific insert phantom generated using a 3D printer for investigations of cardiac computed tomography protocols. Journal of Medical Radiation Sciences, 65(3), pp.175-183.

3. Leng, S., McGee, K., Morris, J., Alexander, A., Kuhlmann, J., Vrieze, T., McCollough, C.H. and Matsumoto, J., 2017. Anatomic modeling using 3D printing: Quality assurance and optimization. 3D Printing in Medicine, 3(6), pp.1-14.

4. Silvestro, E., Betts, K.N., Francavilla, M.L., Andronikou, S. and Sze, R.W., 2020. Imaging properties of additive manufactured (3D printed) materials for potential use for phantom models. Journal of Digital Imaging, 33(2), pp.456464.

5. Mitsouras, D., Liacouras, P., Imanzadeh, A., Giannopoulos, A.A., Cai, T., Kumamaru, K.K., George, E., Wake, N., Caterson, E.J., Pomahac, B., Ho, V.B., Grant, G.T. and Rybicki, F.J., 2015. Medical 3D printing for the radiologist. Radiographics, 35(7), pp.1965-1988.

6. Ho, D., Squelch, A. and Sun, Z., 2017. Modelling of aortic aneurysm and aortic dissection through 3D printing. Journal of Medical Radiation Sciences, 64(1), pp.10-17.

7. Lau, I. and Sun, Z., 2018. Three-dimensional printing in congenital heart disease: A systematic review. Journal of Medical Radiation Sciences, 65(3), pp.226-236.

8. Yea, J.W., Park, J.W., Kim, S.K., Kim, D.Y., Kim, J.G., Seo, C.Y., Jeong, W.H., Jeong, M.Y. and Oh, S.A., 2017. Feasibility of a 3D-printed anthropomorphic patient-specific head phantom for patient-specific quality assurance of intensity-modulated radiotherapy. PLoS One, 12(7), p.e0181560.

9. Ehler, E.D., Barney, B.M., Higgins, P.D. and Dusenbery, K.E., 2014. Patient specific 3D printed phantom for IMRT quality assurance. Physics in Medicine \& Biology, 59(19), pp.5763-5773.

10. Kim, M., Lee, S., Lee, M., Sohn, J.W., Yun, H.G., Choi, J.Y., Jeon, S.W. and Suh, T.S., 2017. Characterization of 3D printing techniques: Toward patient specific quality assurance spine-shaped phantom for stereotactic body radiation therapy. PLoS One, 12(5), p.e0176227.

11. Irnstorfer, N., Unger, E., Hojreh, A. and Homolka, P., 2019. An anthropomorphic phantom representing a prematurely born neonate for digital X-ray imaging using 3D printing: Proof of concept and comparison of image quality from different systems. Scientific Reports, 9(1), p.14357.

12. Anwari, V., Laia, A., Sajjab, S., Ursania, A., Regoc, K. and Pauld, N.S., 2018. Can MRT practice advance 3D printing? Journal of Medical Imaging and Radiation Sciences, 49(3), pp.S12-S13.

13. Anwari, V., 2019. 3D printing in clinical radiology: The MRT experience. Journal of Medical Imaging and Radiation Sciences, 50(3), p.S1.

14. Ikejimba, L.C., Graff, C.G., Rosenthal, S., Badal, A., Ghammraoui, B., Lo, J.Y. and Glick, S.J., 2017. A novel physical anthropomorphic breast phantom for 2D and 3D X-ray imaging. Medical Physics, 44(2), pp.407-416.

15. Jahnke, P., Schwarz, S., Ziegert, M., Schwarz, F.B., Hamm, B. and Scheel, M., 2019. Paper-based 3D printing of anthropomorphic CT phantoms: Feasibility of two construction techniques. European Radiology, 29(3), pp.1384-1390.

16. Solomon, J. and Samei, E., 2014. Quantum noise properties of CT images with anatomical textured backgrounds across reconstruction algorithms: FBP and SAFIRE. Medical Physics, 41(9), p.091908.

17. Leng, S., Chen, B., Vrieze, T., Kuhlmann, J., Yu, L., Alexander, A., Matsumoto, J., Morris, J. and McCollough, C.H., 2016. Construction of realistic phantoms from patient images and a commercial three-dimensional printer. Journal of Medical Imaging, 3(3), p.033501.

18. Jahnke, P., Limberg, F.R.P., Gerbl, A., Ardila Pardo, G.L., Braun, V.P.B., Hamm, B. and Scheel, M., 2017. Radiopaque three-dimensional printing: A method to create realistic CT phantoms. Radiology, 282(2), pp.569-575.

19. Smith-Bindman, R., Wang, Y., Yellen-Nelson, T.R., Moghadassi, M., Wilson, N., Gould, R., Seibert, A., Boone, J.M., Krishnam, M., Lamba, R., Hall, D.J. and Miglioretti, D.L., 2017. Predictors of CT radiation dose and their effect on patient care: A comprehensive analysis using automated data. Radiology, 282(1), pp.182-193. 
20. Al Mahrooqi, K.M.S., Ng, C.K.C. and Sun, Z., 2015. Pediatric computed tomography dose optimization strategies: A literature review. Journal of Medical Imaging and Radiation Sciences, 46(2), pp.241-249.

21. Pucci, J.U., Christophe, B.R., Sisti, J.A. and Connolly Jr., E.S., 2017. Threedimensional printing: Technologies, applications, and limitations in neurosurgery. Biotechnology Advances, 35(5), pp.521-529.

22. Dancewicz, O.L., Sylvander, S.R., Markwell, T.S., Crowe, S.B. and Trapp, J.V., 2017. Radiological properties of $3 D$ printed materials in kilovoltage and megavoltage photon beams. Physica Medica, 38, pp.111-118.

23. Husby, T., Høiseth, A., Alho, A. and Rønningen, H., 1989. Rotational strength of the femoral neck. Computed tomography in cadavers. Acta Orthopaedica Scandinavica, 60(3), pp.288-292.

24. Clarke, B., 2008. Normal bone anatomy and physiology. Clinical Journal of the American Society of Nephrology, 3(Suppl. 3), pp.S131-S139.

25. Popp, A.W., Buffat, H., Eberli, U., Lippuner, K., Ernst, M., Richards, R.G., Stadelmann, V.A. and Windolf, M., 2014. Microstructural parameters of bone evaluated using HR-pQCT correlate with the DXA-derived cortical index and the trabecular bone score in a cohort of randomly selected premenopausal women. PLoS One, 9(2), p.e88946.

26. Von Euw, S., Wang, Y., Laurent, G., Drouet, C., Babonneau, F., Nassif, N. and Azaïs, T., 2019. Bone mineral: New insights into its chemical composition. Scientific Reports, 9(1), p.8456.
27. Rothenberg, S., Abdullah, S. and Hirsch, J., 2017. 3D printing prototypes for healthcare professionals: Creating a reciprocating syringe. Journal of Digital Imaging, 30(5), pp.566-571.

28. Zhou, Z., Buchanan, F., Mitchell, C. and Dunne, N., 2014. Printability of calcium phosphate: Calcium sulfate powders for the application of tissue engineered bone scaffolds using the 3D printing technique. Materials Science and Engineering: C, 38, pp.1-10.

29. Madamesila, J., McGeachy, P., Villarreal Barajas, J.E. and Khan, R., 2016. Characterizing $3 D$ printing in the fabrication of variable density phantoms for quality assurance of radiotherapy. Physica Medica, 32(1), pp.242-247.

30. Ceh, J., Youd, T., Mastrovich, Z., Peterson, C., Khan, S., Sasser, T.A., Sander, I.M., Doney, J., Turner, C. and Leevy, W.M., 2017. Bismuth infusion of ABS enables additive manufacturing of complex radiological phantoms and shielding equipment. Sensors, 17(3), p.E459.

31. Adams, F., Qiu, T., Mark, A., Fritz, B., Kramer, L., Schlager, D., Wetterauer, U., Miernik, A. and Fischer, P., 2017. Soft 3D-printed phantom of the human kidney with collecting system. Annals of Biomedical Engineering, 45(4), pp.963972.

32. Corcione, C.E., Gervaso, F., Scalera, F., Montagna, F., Sannino, A and Maffezzoli, A., 2017. The feasibility of printing polylactic acidnanohydroxyapatite composites using a low-cost fused deposition modeling 3D printer. Journal of Applied Polymer Science, 134(13), pp.1-10.

Received: 29 June 2020. Accepted: 1 August 2020. 